\title{
Hepatobiliary Imaging*
}

\author{
Maggie Doonan \\ Nuclear Diagnostic Products, Inc., Rockaway, New Jersey
}

CE credit: For CE credit, you can access the test for this article, as well as additional JNMT CE tests, online at https://www.snmmilearningcenter.org. Complete the test online no later than December 2023. Your online test will be scored immediately. You may make 3 attempts to pass the test and must answer $80 \%$ of the questions correctly to receive $1.0 \mathrm{CEH}$ (Continuing Education Hour) credit. SNMMI members will have their CEH credit added to their VOICE transcript automatically; nonmembers will be able to print out a CE certificate upon successfully completing the test. The online test is free to SNMMI members; nonmembers must pay $\$ 15.00$ by credit card when logging onto the website to take the test.

\section{H}

epatobiliary scintigraphy is a diagnostic imaging study that uses a radioactive tracer to evaluate hepatocellular function and the biliary system. The production and flow of bile is tracked from the liver as it passes through the biliary system into the small intestine. It is easy, rapid, noninvasive, safe to perform and provides functional and anatomical information about many hepatobiliary disorders.

The origins of functional hepatobiliary testing can be traced back to 1923 when a halogenated fluorescein dye called rose bengal was first used for liver function tests. The first mention of rose bengal is found in Basel, Switzerland, where in 1882 a German patent was granted for a new family of dyes for wool. Agarwala and Wachter reported that the name rose bengal derives from the fact that the dye has a rosy red color. This red color is similar to that of the middle-of-the-forehead dot that indicates marriage in women of the Bengali region of India.

Rose bengal was first labeled with iodine-131 $\left({ }^{131} \mathrm{I}\right)$ in 1955 by Taplin, Meredith, and Kade. A tracing of its pathway in the body was monitored by external gamma ray scintillation counting techniques. By using strategically placed gamma probes, a time-activity curve was produced that helped to differentiate hepatocellular disease from partial common duct obstruction. This counting method evolved into functional imaging on rectilinear and scintillation cameras. For many years, this proved to be sensitive and reliable for detecting liver function and biliary tract obstruction. ${ }^{131} \mathrm{I}-$ rose bengal and later ${ }^{123}$ I-rose bengal were widely utilized despite having limitations in clearance and poor imaging characteristics.

The first announcement of a new hepatobiliary imaging agent can be found forty years ago in an abstract from the $22^{\text {nd }}$ annual Society of Nuclear Medicine meeting, which took

\footnotetext{
For correspondence or reprints contact: Maggie Doonan, Nuclear Diagnostic Products, Inc., 101 Roundhill Dr., Rockaway, NJ 07866.

E-mail: maggiedoonan@aol.com

${ }^{*}$ Reprinted from Bolus N, Farrell MB, Zimmerman J, eds. Abdominal Imaging 2017: Quality, Safety, and Dose Optimization. Society of Nuclear Medicine and Molecular Imaging. 2017:5-21.

COPYRIGHT @ 2020 by the Society of Nuclear Medicine and Molecular Imaging. DOI: $10.2967 /$ jnmt.120.257436
}

place in Philadelphia, PA, when Harvey, Loberg, and Cooper first referred to a new radiopharmaceutical for hepatobiliary imaging labeled with technetium-99m $\left({ }^{99 \mathrm{~m} T c}\right)$. They referred to this new radiopharmaceutical as hepatoiminodiacetic acid, or "HIDA." The term HIDA is now used synonymously with cholescintigraphy and hepatobiliary scintigraphy to mean imaging of the hepatobiliary system using a radiopharmaceutical (Figure 1).

${ }^{99 m}$ Tc-HIDA was an $\mathrm{N}$-substituted iminodiacetic acid structurally related to lidocaine. The first ${ }^{99 \mathrm{~m}} \mathrm{Tc}-\mathrm{HIDA}$ radiopharmaceutical was approved by the Food and Drug Administration in 1982. ${ }^{99 \mathrm{~m} T c-d i m e t h y l ~ i m i n o d i a c e t i c ~ a c i d ~}$ (lidofenin [Technescan: Mallinckrodt]) was a vast improvement over rose bengal, but the quality of the images and diagnostic utility were suboptimal in patients with serum bilirubin levels above $5.0 \mathrm{mg} / \mathrm{dl}$, and therefore, it is no longer commercially available. Numerous other hepatobiliary radiopharmaceuticals were developed, and each had different rates of hepatic uptake and clearance. Lidocaine analogs such as paraisopropyl iminodiacetic acid, bromotriethyl iminodiacetic acid, diethyl iminodiacetic acid, and diisopropyl iminodiacetic acid were evaluated. The Food and Drug Administration approved 99mTc-disofenin (2, 6-diisopropylacetanilido iminodiacetic acid, [Hepatolite: Pharmalucence]) in 1992, and in 1993, 99m Tc-mebrofenin (bromo2, 4, 6-trimethylacetanilido iminodiacetic acid [Choletec: Bracco]) was approved. Both ${ }^{99 m} \mathrm{Tc}-$ disofenin and ${ }^{99 \mathrm{~m}} \mathrm{Tc}-\mathrm{mebrofenin}$ are available and commonly used today.

\section{RATIONALE/INDICATIONS/CONTRAINDICATIONS}

Gallstone disease is common and accounts for greater than 700,000 cholecystectomies and costs of between five and six billion dollars annually in the United States. Risk factors that cannot be changed include female gender, increasing age, and genetic traits. Other risk factors are obesity, metabolic syndrome, rapid weight loss, diseases such as cirrhosis and Crohn's disease, and gallbladder stasis. A high caloric diet is also an established risk. The role of nuclear medicine in the evaluation of hepatobiliary disease increased with the development of the ${ }^{99 \mathrm{~m}} \mathrm{Tc}$ hepatobiliary agents. Prior to 1982, the common diagnostic choices included oral cholecystograms 


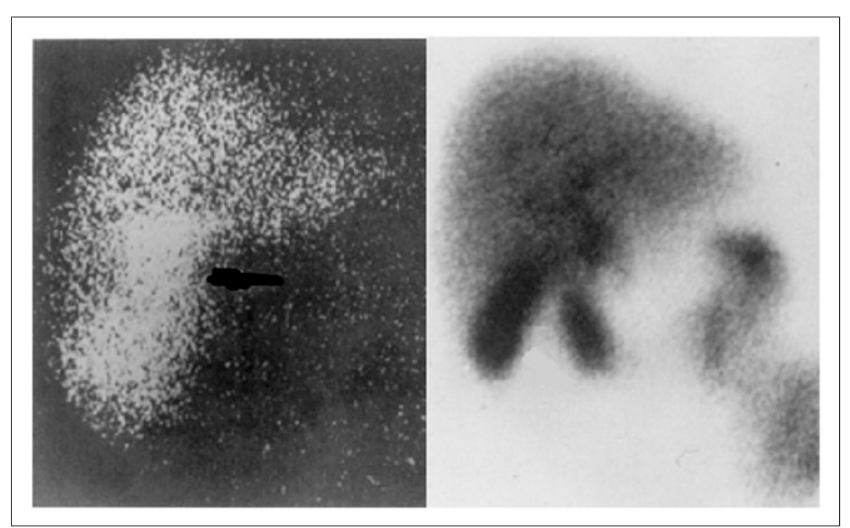

FIGURE 1. Early ${ }^{123}$-rose bengal images (left) compared to 99mTc-HIDA images (right). This image was originally published in JNMT. Ziessman. Hepatobiliary Scintigraphy. J Nucl Med Technol. 2014;42:250. (C) SNMMI.

and intravenous cholangiograms, which both utilized iodinated contrast agents. There were many side effects with the iodinated contrast, whether given orally or intravenously. Frequently seen side effects included nausea, vomiting, and diarrhea. More severe side effects—-such as bronchospasm, laryngospasm, hypotension, renal failure, pulmonary edema, and acute anaphylaxis - were rare. Ultrasonography remains part of the initial workup for suspected hepatobiliary disease and is a quick, noninvasive, nonfunctional anatomic exam that does not use ionizing radiation.

Hepatobiliary scintigraphy evaluates function by following the path of a tracer through the hepatobiliary system. The radiopharmaceutical is transported through the blood stream bound to serum albumin. Being tightly bound to a protein minimizes clearance by the kidneys. The tracer is extracted by the liver hepatocytes and is not metabolized. The hepatocytes excrete the tracer as bile into the canaliculi, where it drains into the many bile ducts within the liver (Figure 2). The bile ducts (biliary tree) join to form the right and left hepatic ducts, which collect bile from the right and left lobes of the liver. The right and left hepatic ducts join to form the common hepatic duct, which drains all bile from

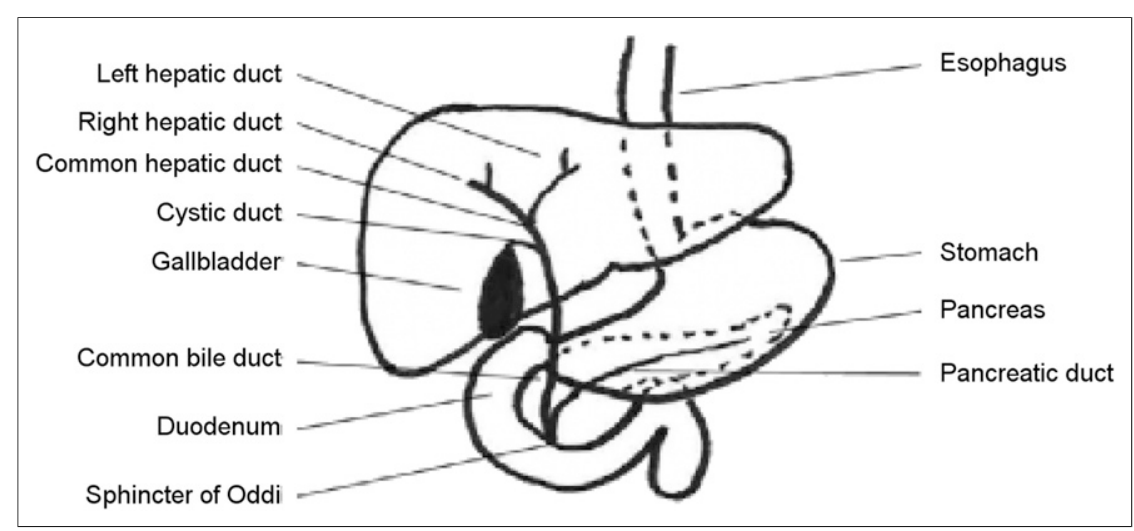

FIGURE 2. Diagram of the hepatobiliary system. the liver. The common hepatic duct joins with the cystic duct coming from the gallbladder to form the common bile duct, which empties into the duodenum of the small intestine.

Bile flows slowly when the patient is in the fasting state. About half of the bile produced then drains directly into the common bile duct, and the other half is diverted through the cystic duct into the gallbladder for storage. When food enters the small intestine, the gallbladder contracts and the sphincter of Oddi relaxes and opens. Bile then flows from the gallbladder into the small intestine to aid in digestion.

When optimally performed, hepatobiliary scintigraphy is a sensitive method for detecting many disorders involving the liver and biliary system. Indications for hepatobiliary scanning include, but are not limited to: functional biliary pain syndromes in adults, functional biliary pain syndromes in the pediatric population, acute cholecystitis, right upper quadrant pain, biliary system patency, bile leakage, neonatal hyperbilirubinemia (biliary atresia versus neonatal hepatitis syndrome), assessment of a biliary enteric bypass (e.g., Kasai procedure), assessment of a liver transplant, afferent loop syndrome, assessment of choledochal cysts, assessment of biliary stent patency, calculation of the gallbladder ejection fraction, functional assessment of the liver before partial hepatectomy, demonstration of anomalous liver lobulation, enterogastric (duodenogastric) reflux assessment, esophageal bile reflux after gastrectomy, sphincter of Oddi dysfunction, evaluation of hepatocyte tumors, and enterogastric bile reflux.

The only contraindication for hepatobiliary imaging related to the radiopharmaceutical is hypersensitivity to a hepatobiliary compound. There is a warning that it is theoretically possible for allergic reactions to develop in patients who receive multiple doses of a hepatobiliary compound. Other contraindications are related to the medications used in the interventions, such as sincalide, which is contraindicated in patients with intestinal obstruction or hypersensitivity to sincalide. In addition, morphine is contraindicated in children who have increased intracranial pressure, non-ventilated patients with respiratory depression, and individuals with morphine allergy. Acute pancreatitis is also a relative contraindication.

\section{PATIENT PREPARATION/ EDUCATION/FOOD/MEDICATION RESTRICTIONS}

Adult patients should have nothing by mouth for a minimum of 2 hours-and preferably 6 hours-before the injection of the radiopharmaceutical. Pain medications should be withheld. If the patient is post-cholecystectomy and being evaluated for bile leak, the patient does not need to fast or have pain medications withheld. 
Infants are usually evaluated for biliary atresia and do not need to fast, as biliary-to-bowel transit is the endpoint. If imaged for other indications, children should fast for 2 to 4 hours; infants for only 2 hours. If medically necessary, infants may have clear liquids.

If a patient has been fasting for greater than 24 hours or is receiving total parenteral nutrition, pretreatment with sincalide is recommended. Extended fasting may cause delayed filling of the gallbladder. Sincalide is administered before the radiopharmaceutical injection to empty the gallbladder of concentrated bile. The pharmacologic dose $(0.02 \mathrm{ug} / \mathrm{kg})$ should be infused over a period of 60 minutes for optimal gallbladder contraction, and the radiopharmaceutical should be administered at least 30 minutes later. Adherence to the above recommendations is necessary to avoid false-positive non-visualization of the gallbladder.

The patient history should include current medications, especially opioids, as they can inhibit gallbladder contraction, which is evidenced by delayed biliary to bowel transit. The patient's prior surgical history should also be documented.

\section{IMAGING PROCEDURE}

The non-jaundiced patient is injected intravenously with 111 to $185 \mathrm{MBq}(3-5 \mathrm{mCi})$ of either ${ }^{99 \mathrm{~m}} \mathrm{Tc}$-disofenin or ${ }^{99 \mathrm{~m}} \mathrm{Tc}$-mebrofenin. If the patient has hyperbilirubinemia, a higher administered dose may be needed. Mebrofenin has higher hepatic extraction and may be preferred in moderate to severe hepatic dysfunction.

The dose for infants and children is $1.8 \mathrm{MBq} / \mathrm{kg}(0.05$ $\mathrm{mCi} / \mathrm{kg})$. The minimum administered activity is $18.5 \mathrm{MBq}$ $(0.5 \mathrm{mCi})$. In neonates with hyperbilirubinemia, mebrofenin is preferred with a minimum administered dose of $37 \mathrm{MBq}$ (1.0 mCi), because 24-hour delayed images are usually necessary.

The patient position should be supine, with the liver in the upper quadrant of the field of view, and the camera head should be anterior at 180 degrees. Patients with severe pain while lying supine may benefit from being allowed to turn on their side between images.

The suggested parameters for hepatobiliary scintigraphy include a large-field-of-view gamma camera able to encompass the whole abdomen in the field of view. A low-energy allpurpose or high-resolution collimator is used. The energy window photopeak is set at $140 \mathrm{keV}$, with a $20 \%$ energy window. A $128 \times 128$ image matrix should be used. Additional 500k static views may be required.

Dynamic images (1 min/frame) or sequential static $(500 \mathrm{~K}$ counts) of the liver, biliary tree, and abdomen are obtained for 1 hour. Dynamic imaging is preferred and should be performed whenever a bile leak is suspected or a timeactivity curve is desired, as well as to calculate the gallbladder ejection fraction $(\mathrm{GBEF})$.

An optional 60-second blood flow phase may be done. The blood flow phase may show increased flow to the gallbladder fossa such as is seen in severe cases of acute cholecystitis or in other causes of intraabdominal pain that may be caused by infection or vascular tumors.

Following the 60-minute dynamic imaging, additional images may be required for up to 4 hours if the gallbladder is not visualized at 1 hour, if common duct obstruction is suspected, or if biliary atresia is suspected. If biliary leak is suspected, delayed imaging and SPECT/CT are useful. Delayed imaging at 18 to 24 hours may also be necessary. Additional images in the right anterior oblique or right lateral views are helpful for separating the common bile duct and gallbladder from the duodenum and the kidneys. Visualization of the kidneys may be due to an alternative excretory pathway when there is hepatic failure and increased serum bilirubin levels.

\section{Radiation Exposure}

The ICRP 106 model estimates that IV injection of $148 \mathrm{MBq}$ (4 mCi) ${ }^{99 m} \mathrm{Tc}$-disofenin/mebrofenin for a hepatobiliary study would impart an approximate effective dose of $2.5 \mathrm{mSv}$ $(0.25 \mathrm{rem})$ in an adult male. The critical organ for this study is the gallbladder, which would receive $16.3 \mathrm{mGy}$ (1.63 $\mathrm{rad})$. Intravenous injection for an adult female of $148 \mathrm{MBq}$ (4 mCi) ${ }^{99} \mathrm{~m}$ Tc-disofenin/mebrofenin would impart an approximate effective dose of $3.1 \mathrm{mSv}(0.31 \mathrm{rem})$. The critical organ for this study is the gallbladder, which would receive $17.8 \mathrm{mGy}(1.78 \mathrm{rad})$.

\section{INTERVENTIONS}

Several pharmacologic interventions to hepatobiliary scintigraphy may be required to improve the diagnostic accuracy of the test.

\section{Morphine Sulfate}

When the gallbladder is not visualized within 30 to 60 minutes and acute cholecystitis is suspected, morphine is indicated. Morphine causes a temporary spasm of the sphincter of Oddi, which increases intrabiliary pressure, and if the cystic duct is patent, bile can then flow into the gallbladder. For best results, tracer should be present in the small bowel and the intrahepatic biliary tree and common bile duct. A weight-based dose of $0.04 \mathrm{mg} / \mathrm{kg}$ or a standard dose of $2 \mathrm{mg}$ is administered intravenously over 2 to 3 minutes. If there is little activity left in the liver, an additional $111 \mathrm{MBq}(3 \mathrm{mCi})$ radiotracer may be injected intravenously before injection of the morphine. Imaging continues for an additional 30 to 60 minutes after morphine administration. Unless the cystic duct is obstructed, the gallbladder is usually seen by 30 to 60 minutes. When morphine is administered, images at 4 hours are usually unnecessary, which is beneficial for acutely ill patients.

\section{Sincalide Stimulation}

Sincalide simulates cholecystokinin, which causes gallbladder contraction, and relaxation of the sphincter of Oddi can be used to assess gallbladder contractility and GBEF. Following visualization of the gallbladder, sincalide is infused at $0.02 \mu \mathrm{g} /$ $\mathrm{kg}$ over 60 minutes. The GBEF determined using this technique should be greater than or equal to $35 \%$ to $40 \%$. 


\section{Phenobarbital}

In cases where biliary atresia is suspected, pretreatment with oral phenobarbital, $5 \mathrm{mg} / \mathrm{kg}$ per day divided into two doses daily, for a minimum of 3 to 5 days before hepatobiliary imaging, increases bile flow and biliary excretion of the radiopharmaceutical.

\section{PROCESSING}

Upon completion of the acquisition, the suggested mode for display is serial static images for the total length of the study. In particular, cine display is desirable when a bile leak is suspected. The dynamic images may also be reformatted to 4-to-6-minute images for display digitally or on film.

When reviewing the images, shielding of the gut may allow for better visualization of a low-count gallbladder. The use of motion correction software is recommended if there is movement during a dynamic acquisition to calculate the GBEF.

\section{Gallbladder Ejection Fraction}

To calculate a GBEF, a region of interest is drawn around the full gallbladder in the last image immediately before administration of the sincalide. A background region is drawn over the adjacent liver, taking care to exclude ductal activity. The GBEF is calculated from the time-activity curve using the formula [(net maximum gallbladder counts - net minimum gallbladder counts)/net maximum gallbladder counts] $\times 100$, corrected for background and radioactive decay (Figure 3 ).

\section{IMAGE INTERPRETATION}

\section{Normal Results}

The normal hepatobiliary scan demonstrates concentration of the tracer in the liver within a few minutes. Most of the tracer has been removed from the bloodstream and concentrated in the liver within 15 to 30 minutes post-injection. The tracer then begins to concentrate in the biliary ducts and gallbladder, with the gallbladder usually visualized by 45 to 60 minutes. The left and right hepatic bile ducts are usually seen, along with the common hepatic duct and common bile duct. Visualization of the gallbladder later than 30

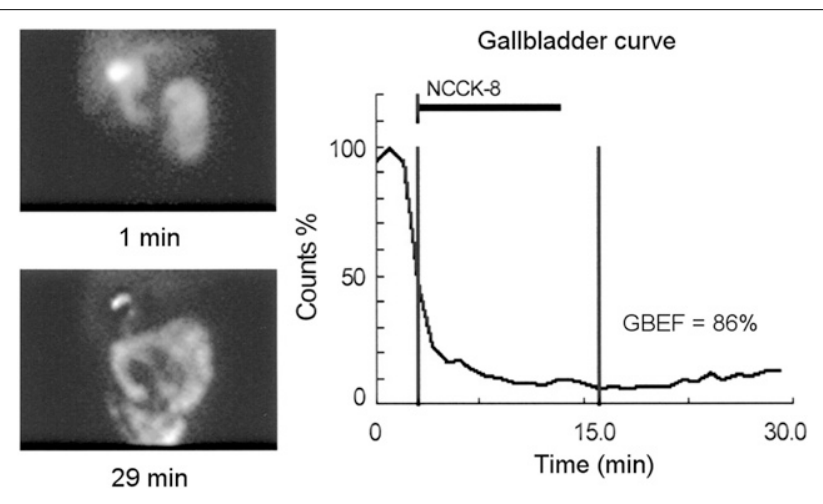

FIGURE 3. Normal gallbladder ejection fraction greater than $35 \%$ following the administration of CCK. This image was originally published in JNM. Krishnamurthy S. Comparison of Gallbladder Function Obtained with Regular CCK-8 and Pharmacy-Compounded CCK-8. J Nucl Med. 2008;44(4);499-504. (c) SNMMI.

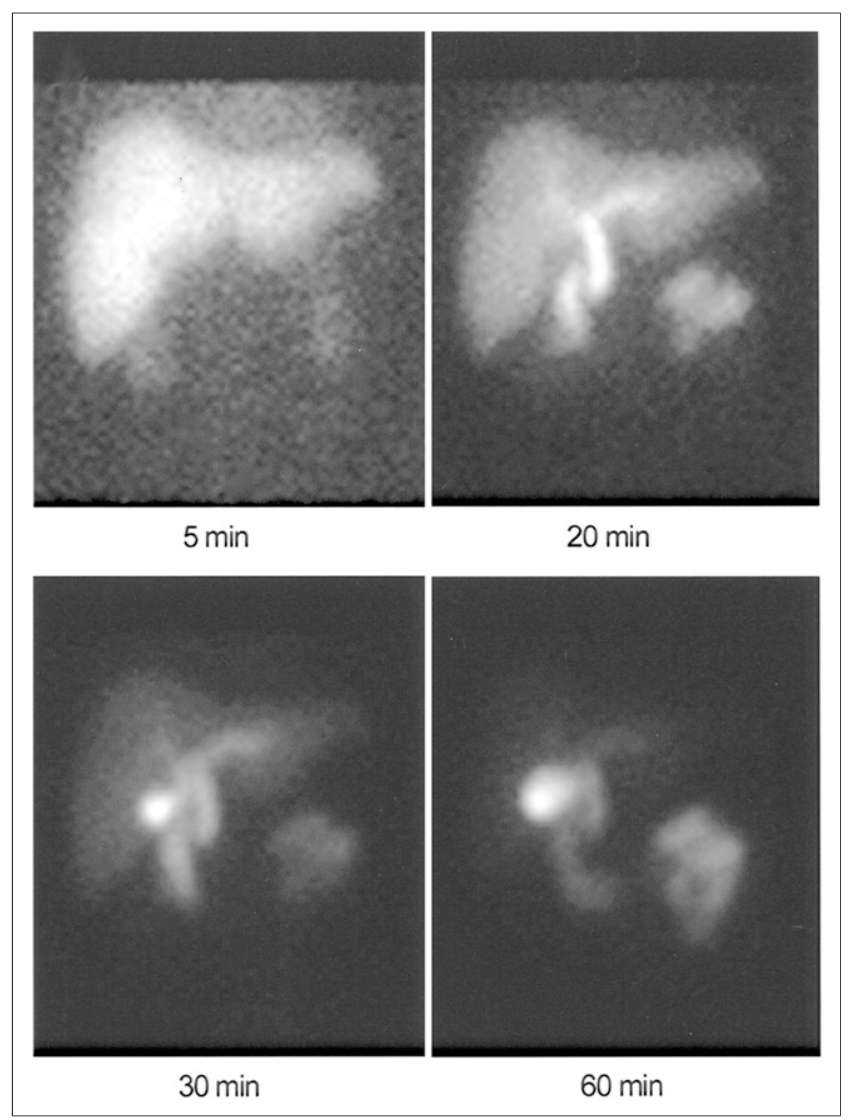

FIGURE 4. Normal hepatobiliary imaging demonstrating immediate uptake of the tracer within the liver and appearance of the gallbladder by 30 minutes. The right and left hepatic ducts, common hepatic duct, and common bile duct are visualized. There is visualization of tracer within the gastrointestinal tract within 30 minutes. This image was originally published in JNM. Krishnamurthy S. Comparison of Gallbladder Function Obtained with Regular CCK-8 and Pharmacy-Compounded CCK-8. J Nucl Med. 2008;44(4);499-504. (c) SNMMI.

to 60 minutes after administration of the radiopharmaceutical is considered delayed. The radiopharmaceutical should be seen in the gastrointestinal tract by 30 minutes. GBEF should be greater than or equal to $35 \%$ to $40 \%$ if the standardized sincalide infusion protocol is used (Figure 4).

\section{ABNORMAL RESULTS}

\section{Acute Cholecystitis}

Acute cholecystitis is inflammation of the gallbladder due to blockage of the cystic duct by a gallstone (Figure 5).

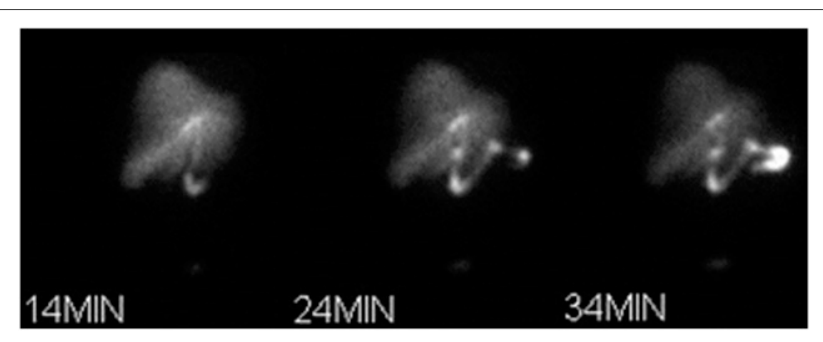

FIGURE 5. Acute cholecystitis. 
The blockage of the duct causes bile to build up, increasing pressure within the gallbladder and causing irritation. Swelling and infection may occur. Symptoms include right upper quadrant pain, nausea, and vomiting.

Scintigraphy demonstrates good hepatic function and common bile duct excretion to the small bowel with nonvisualization of the gallbladder. Non-visualization of the gallbladder 3 to 4 hours after administration of the radiopharmaceutical or within 30 minutes of morphine administration is indicative of acute cholecystitis.

Acute acalculous cholecystitis is an inflammation of the gallbladder without the presence of gallstones. It is an uncommon but life-threatening disease that is often a complication of other medical or surgical conditions. Reduced GBEF after administration of sincalide $(<40 \%)$ is indicative of acute cholecystitis.

\section{Chronic Cholecystitis}

Chronic cholecystitis is the most common reason for gallbladder visualization more than 60 minutes post-injection. Chronic cholecystitis results from damage to the gallbladder from repeated acute inflammation from gallstones where the gallbladder walls become thick and scarred. As a result, the gallbladder shrinks in size. Symptoms often occur after a fatty meal and include abdominal pain, cramping, and bloating that can extend to the right scapula accompanied by fever, chills, nausea, and vomiting. Treatment is usually cholecystectomy.

On hepatobiliary imaging, the gallbladder is typically seen 3 to 4 hours after administration of the radiopharmaceutical or within 30 to 60 minutes after morphine administration (Figure 6). If the bowel is visualized but the gallbladder is

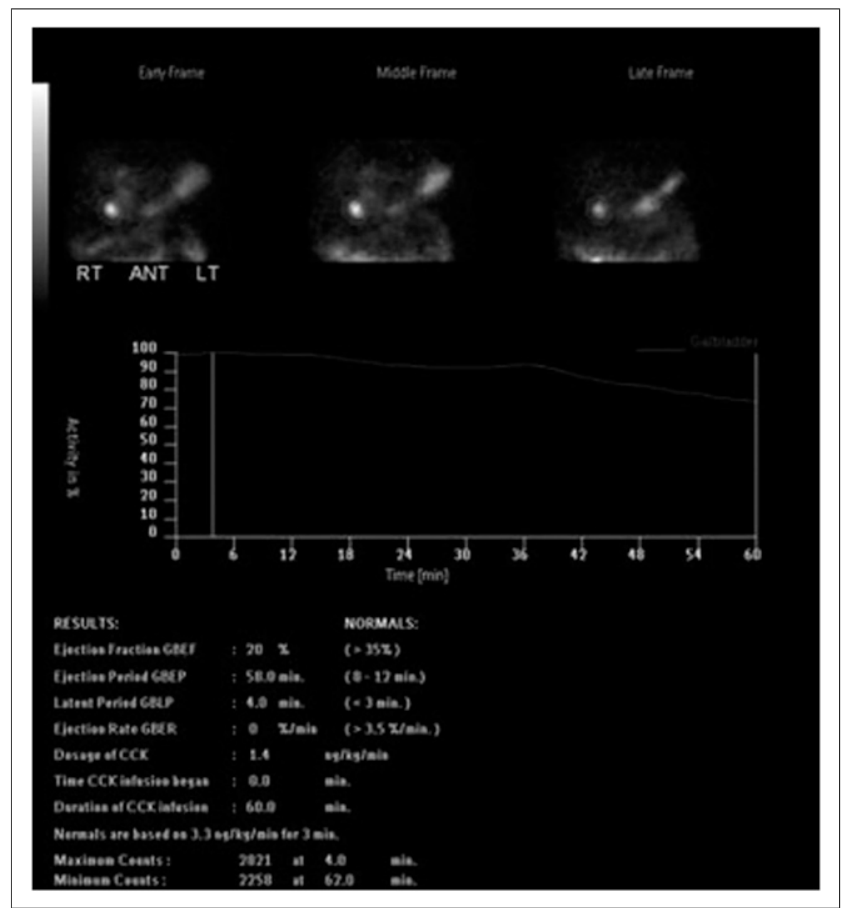

FIGURE 6. Chronic cholecystitis.

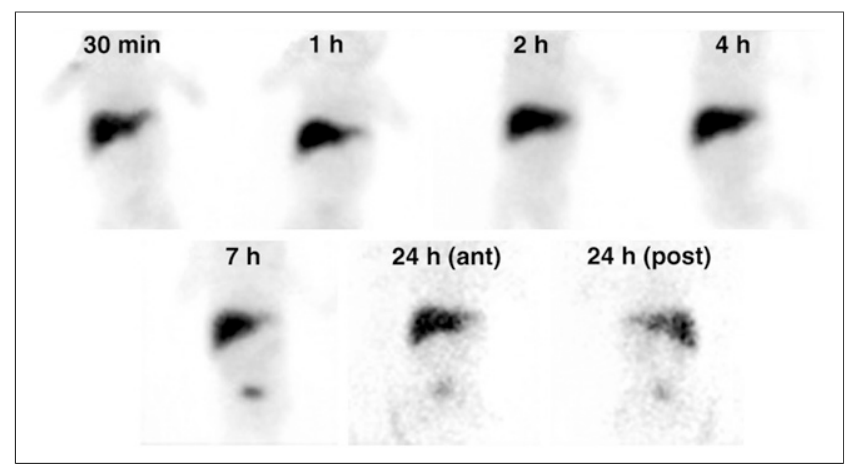

FIGURE 7. Hepatobiliary imaging of a 45-day-old infant with jaundice using 99mTc-mebrofenin. The images demonstrate good uptake of the tracer in the liver and bowel and nonvisualization of the biliary tree and intestines. This image was originally published in JNM. Poddar U. Ursodeoxycholic Acid-Augmented Hepatobiliary Scintigraphy in the Evaluation of Neonatal Jaundice. J Nucl Med. 2004;45(9):1488. (c) SNMMI.

not visualized until 3 to 4 hours later, that is consistent with chronic cholecystitis.

Acalculous chronic cholecystitis is a form of chronic cholecystitis where gallstones are not present. Other than that, it is clinically indistinguishable from chronic cholecystitis. Hepatobiliary imaging with sincalide is used to diagnose chronic acalculous cholecystitis, and the GBEF is below normal.

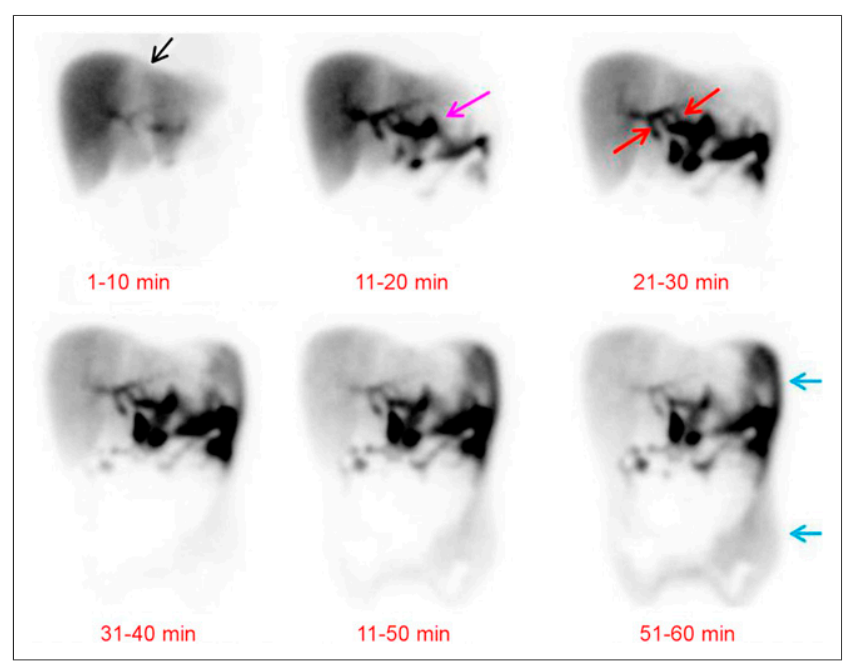

FIGURE 8. Hepatobiliary imaging over 60 minutes demonstrating a patient with a known hepatic laceration between the two hepatic lobes that was diagnosed by CT scan (black arrow). The images demonstrate increasing tracer accumulating outside of the biliary system (pink arrow). The red arrows show lines of tracer uptake that connect to an abnormal focus of tracer to the left of the intrahepatic duct. Over time, the abnormal tracer spreads in the paracolic gutters and sometimes throughout the abdomen, as noted by the blue arrows. These findings are indicative of a bile leak at left intrahepatic duct. This image was originally published in JNMT. Naeem S. Precise Localization of a Bile Leak with Hepatobiliary Scintigraphy. J Nucl Med Technol. 2015;44:44. (c) SNMMI. 


\section{Biliary Atresia}

Biliary atresia is a rare infant disease caused from bile ducts that did not form properly during pregnancy or because of damage to the bile ducts due to an immune response to a viral infection. If bile flows from the liver to the gallbladder, it causes damage and scarring of the liver cells and eventually liver failure. Symptoms of jaundice-dark urine, clay-colored stools, weight loss, and irritability-usually develop 2 to 8 weeks after birth.

Hepatobiliary imaging shows persistent uptake of the tracer within the liver, with no biliary to bowel transit for up to 24 hours (Figure 7).

\section{Bile Leak}

Bile leaks typically occur following cholecystectomy or other surgery of the biliary tract. Laparoscopic cholecystectomy has a higher rate of occurrence of bile leaks compared to open cholecystectomy surgery. Hepatobiliary imaging demonstrates increasing accumulation of the tracer in the area of the gallbladder fossa, spreading to other areas of the abdomen. A biliary leak is diagnosed when the tracer is found in a location other than the liver, gallbladder, bile ducts, bowel, or urine (Figure 8).

\section{SENSITIVITY/SPECIFICITY/ACCURACY}

Hepatobiliary imaging has a sensitivity of $95 \%$ to $98 \%$ and specificity of $90 \%$ for acute cholecystitis. Comparisons of hepatobiliary imaging with sonography have found that hepatobiliary scans are more sensitive for early acute cholecystitis than ultrasound. Sonography is very good at documenting the presence of gallstones, with a $95 \%$ sensitivity for stones larger than $2 \mathrm{~mm}$. The sensitivity of hepatobiliary imaging for acute acalculous cholecystitis is lower than for acute calculous cholecystitis, at approximately $80 \%$.

\section{ARTIFACTS AND OTHER FINDINGS}

There are a number of sources of error in hepatobiliary imaging. False-positive studies such as nonvisualization of the gallbladder in the absence of acute cholecystitis can result from patient fasting less than 2 to 4 hours or from prolonged fasting greater than 24 hours, especially if the patient is receiving total parenteral nutrition. Severe hepatocellular disease, high-grade common bile duct obstruction, pancreatitis, severe chronic cholecystitis, and previous cholecystectomy can also cause false-positive scans. False-negative scans where the gallbladder is visualized in acute cholecystitis are rare but may be caused by a loop of bowel mimicking the gallbladder, acute acalculous cholecystitis, bile leak from gallbladder perforation, or congenital abnormalities.

Continuous monitoring of the serial images is necessary so that appropriate additional images, or interventions, may occur while the ${ }^{99 \mathrm{~m}} \mathrm{Tc}$ hepatobiliary imaging agent is still present in the liver for sufficient count rates.

\section{Rim Sign}

Increased uptake of the radiotracer surrounding the gallbladder fossa is referred to as the "rim sign." This is
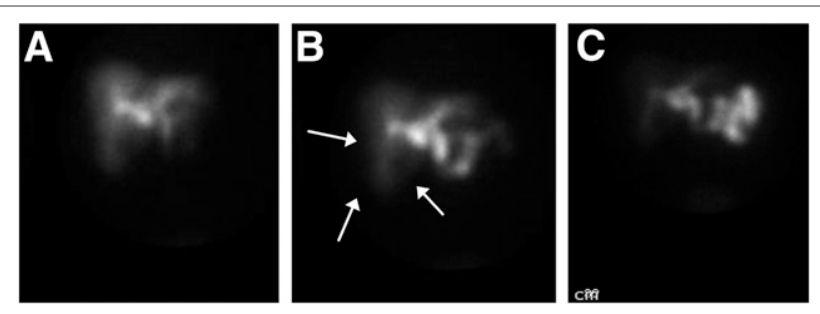

FIGURE 9. Hepatobiliary imaging of the "Rim Sign" (arrows) at (a) 20 minutes, (b) 30 minutes, and (c) 60 minutes. This image was originally published in JNM. Rodrique P. Gallbladder Fossa Abscess Masquerading as Cholecystitis After Cholecystectomy. J Nucl Med Technol. 2015;43. (c) SNMMI.

an ancillary finding in about $25 \%$ to $35 \%$ of patients with acute cholecystitis. The rim sign is caused by severe gallbladder inflammation extending to the adjacent liver that results in increased blood flow to the region (Figure 9). In particular, the rim sign has been associated with gangrene and perforation. The rim sign has very high specificity $(\sim 100 \%)$ and positive predictive value $(\sim 100 \%)$ for complicated acute cholecystitis but with low sensitivity of $49 \%$ and low negative predictive value of $39 \%$.

\section{Cystic Duct Sign}

A "cystic duct sign" (also called the "nubbin sign") occurs when there is dilation of the cystic duct, and the images display a focal area of activity medial to the usual position of the gallbladder (Figure 10). This focal area of activity remains constant over time and can be misinterpreted as the gallbladder. A SPECT/CT is helpful in making the diagnosis.

\section{Bile Stasis}

Stasis of radioactivity within the duodenal bulb may mimic gallbladder filling (Figure 11). Oral administration of 100 to $200 \mathrm{~mL}$ of water, if not contraindicated for other reasons, can help to wash out of the duodenal loop if stasis of bile is overlying the gallbladder fossa. Lateral and oblique views can help to demonstrate small bowel and gallbladder definition or separation. A left anterior oblique image will separate the bowel and gallbladder. A right lateral view will

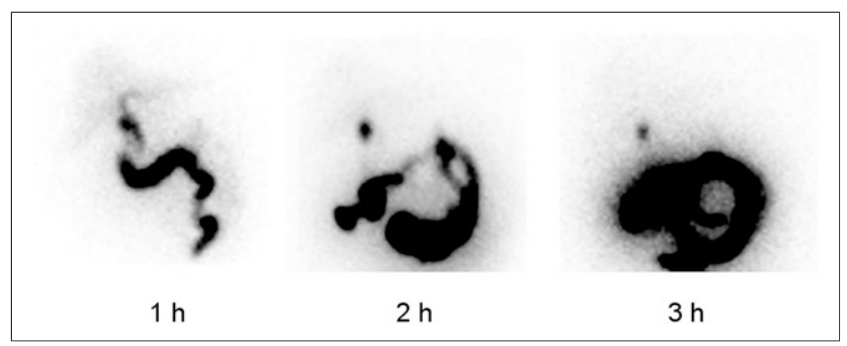

FIGURE 10. Hepatobiliary imaging demonstrating tracer accumulation in the cystic duct that remains unchanged over time consistent with cystic duct sign. This image was originally published in JNM. Ziessman HA. Hepatobiliary Scintigraphy in 2014. J Nucl Med. 2014;55:970. (c) SNMMI. 
FIGURE 11. Retention of tracer within the duodenum mimicking the gallbladder. This image was originally published in JNMT. Bhambhvani P. Cover Image. J Nucl Med Technol. 2012;40(2):Cover Image. (C) SNMMI.

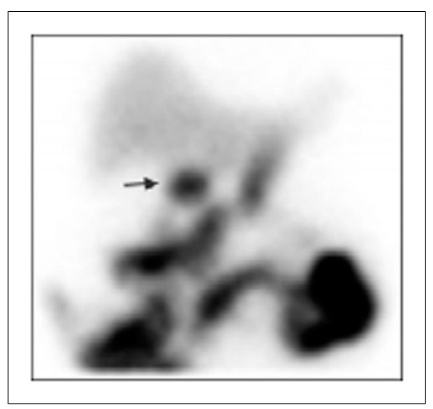

separate the bowel and gallbladder, with the gallbladder being the anterior organ.

\section{SUMMARY}

An aging population, the rising epidemic of obesity, and the existence of metabolic syndrome are certain to perpetuate the frequency and complications of gallstone disease. The prevalence of these conditions makes the continued ability to diagnose gallbladder disease accurately a necessity. ${ }^{99 m}$ Tc hepatobiliary scanning, with its high sensitivity and specificity, has many advantages in diagnosing suspected acute cholecystitis, and it is an ideal diagnostic tool for numerous other functional diseases of the hepatobiliary system.

\section{REFERENCES/SUGGESTED READINGS}

1. Alexander W. Rose bengal: From a wool dye to a cancer therapy. PT. 2010;35:474 478.

2. Ahmed A, Cheung RC, Keeffe EB. Management of gallstones and their complications. Am Fam Physician. 2000;61:1673-1680.

3. Alobaidi M, Gupta R, Jafri SZ, Fink-Bennett DM. Current trends in imaging evaluation of acute cholecystitis. Emerg Radiol. 2004;10(5):256-258.
4. Bracco Diagnostics (2014). Choletec ${ }^{\circledR}$ (Kit for the Preparation of Technetium Tc99m Mebrofenin). Spokane, WA. Retrieved from http://imaging.bracco.com/ sites/braccoimaging.com/files/technica_sheet_pdf/us-en-2016-07-20-spc-choletec_0.pdf.

5. Bracco Diagnostics (2014). Kinevac ${ }^{\circledR}$ (sincalide for Injection). Spokane, WA. Retrieved from http://imaging.bracco.com/us-en/products/nuclear-medicineradiopharmaceuticals/ kinevac.

6. Harvey E, Loberg M, Cooper M. Tc99m-HIDA: A new radiopharmaceutical for hepatic-biliary imaging. J Nucl Med Technol. 1975;16(6):533[abstract].

7. Meekin GK, Ziessman HA, Klappenbach RS (1987). Prognostic value and pathophysiologic significance of the rim sign in cholescintigraphy. J Nucl Med, 1987;28(11):1678-1682.

8. Nordyke R. Metabolic and physiologic aspects of 131 rose bengal in studying liver function. Semin Nucl Med. 1972;2(2):157-66.

9. Pharmalucence (2008). Hepatolite ${ }^{\circledR}$ Kit for the Preparation of Technetium Tc $99 \mathrm{~m}$ Disofenin for Injection. Bedford, MA. Retrieved from http://www.pharmalucence. com/images/HepatoliteInsert.pdf.

10. Shaffer EA. Epidemiology and risk factors for gallstone disease: Has the paradigm changed in the 21st century? Curr Gastroenterol Rep. 2005;7(2):132-40.

11. Society of Nuclear Medicine. The SNM Procedure Guideline for General Imaging 6.0 (2010). Retrieved from http://snmmi.files.cms-plus.com/docs/General_Imaging Version_6.0.pdf.

12. Society of Nuclear Medicine and Molecular Imaging. SNMMI Position Statement on Dose Optimization for Nuclear Medicine and Molecular Imaging Procedures. 2012. Retrieved from http://snmmi.files.cms-plus.com/docs/SNM_Position_Statement_ on_Dose_Optimization_FINAL_June_2012.pdf.

13. Stinton LM, Shaffer EA. Epidemiology of gallbladder disease: Cholelithiasis and Cancer. Gut Liver. 2012;6(2):172-87.

14. Taplin GV, Meredith OM, Kade H. The radioactive (I131 tagged) rose bengal uptake-excretion test for liver function using external gamma-ray scintillation counting techniques. J Lab Clin Med. 1955;45(5):665-678.

15. Tulchinsky M, Ciak BW, Delbeke D, Hilson A, Holes-Lewis KA, Stabin MG, Zeissman HA. SNM Practice Guideline for Hepatobiliary Scintigraphy 4.0. J Nucl Med Technol. 2010;38(4):210-218.

16. Weissman HS, Badia J, Sugarman LA, Kluger L, Rosenblatt R, Freeman LM. Spectrum of 99m-Tc-IDA cholescintigraphic patterns in acute cholecystitis. Radiology. 1981;138(1):167-175.

17. Weissman HS, Sugarman LA, Freeman LM. The role of technetium-99m iminodiacetic acid cholescintigraphy. In: Freeman LM, Weissman HS, eds. Nuclear Medicine Annual. New York: Raven Press, 1981:35-89.

18. Zeissman HA. Hepatobiliary scintigraphy. J Nucl Med Technol. 2014;42(4):249-259.

19. Zeissman HA, Tulchinsky M, Lavely WC, Gaughan, JP, Allen TW, Maru A, Parkman HP. Sincalide-stimulated cholescintigraphy: A multicenter investigation to determine optimal infusion methodology and gallbladder ejection fraction normal values. J Nucl Med. 2010;51(2):277-281. 